\title{
Preparation and electrochemical property of CMC/MWCNT composite using ionic liquid as solvent
}

\author{
ZHAO TingKai ${ }^{1,2 *}$, LIU LeHao ${ }^{1}$, LI GuangMing ${ }^{1}$, DU Li $^{1}$, ZHAO Xing ${ }^{1}$, YAN Jin ${ }^{1}$, \\ CHENG YouLiang ${ }^{1}$, DANG ALei ${ }^{1} \&$ LI TieHu ${ }^{1}$ \\ ${ }^{1}$ School of Materials Science and Engineering, Northwestern Polytechnical University, Xi'an 710072, China; \\ ${ }^{2}$ Department of Materials Science and Engineering, Northwestern University, Evanston 60208, USA
}

Received December 30, 2011; accepted January 19, 2012

\begin{abstract}
Carboxymethyl cellulose sodium (CMC)/multi-walled carbon nanotube (MWCNT) composite was prepared by dissolving CMC with ionic liquid as solvent. The microstructure and electrochemical properties of CMC/MWCNTs were studied using field emission scanning electron microscopy (FE-SEM), high-resolution transmission electron microscopy (HR-TEM), X-ray diffraction (XRD) system and electrochemical workstation. The experimental results indicated that the CMC dissolved with ionic liquid could be uniformly enwrapped on the surface of MWCNTs, and the coating thickness of CMC was about $5.4 \mathrm{~nm}$. There are obvious oxidation peaks in the cyclic voltammograms curves of glassy carbon electrode coated with a CMC/MWCNT composite in $\mathrm{H}_{2} \mathrm{O}_{2}$ phosphate buffer solution. The MWCNT content and ultrasonic time strongly affected the dispersivity and electrochemical property of CMC/MWCNT composite. While the MWCNT content and ultrasonic processing time was $2 \mathrm{wt} \%$ and $2 \mathrm{~h}$ respectively, CMC/MWCNT composite exhibited excellent electrocatalytic activity.
\end{abstract}

multi-walled carbon nanotube, carboxymethyl cellulose sodium, ionic liquid, electrochemical property

Citation: Zhao T K, Liu L H, Li G M, et al. Preparation and electrochemical property of CMC/MWCNT composite using ionic liquid as solvent. Chin Sci Bull, 2012, 57: 1620-1625, doi: 10.1007/s11434-012-5009-2

Since Iijima's landmark paper in 1991 [1], carbon nanotubes (CNTs) have attracted scientists' extensive research due to their remarkable physical and chemical properties, and the potential application in the fields of electronic devices, catalysts and biomedicine. In recent years, the studies of CNTs and biological molecules composite for the purpose of biological applications are rapidly increasing. It has become a new and hot research topic owing to integrating the performances both biologically active molecules (enzymes, proteins, DNA, etc.) and carbon nanotubes $[2,3]$. Cellulose, as an inexhaustible natural and renewable polymer material resource, grows and exists in green plants. In addition, cellulose possesses the merits of low cost, rich resource and good biocompatibility. Therefore, cellulose/ multi-walled carbon nanotube (MWCNT) composite has good potential application in the fields of biomaterials and

\footnotetext{
*Corresponding author (email: ztk-xjtu@163.com; ztk@nwpu.edu.cn)
}

electrochemistry $[4,5]$.

Ionic liquid (IL) as a new type of material, is considered to be an environmentally friendly green solvent. IL has many advantages such as high thermostability, high ionic conductivity, low toxicity, non-inflammability, non-volatility and non-explosive ability. It has the nature of both liquid and ionic solvent compared to the solid and conventional liquid material. And it also has lower melting point (not higher than $100^{\circ} \mathrm{C}$ ) than the molten salt [6-8]. Recently, Swatloski et al. [9] reported that the IL of bromide 1-butyl3-methylimidazolium ([BMIM]Br) which destructed the hydrogen bonds between cellulose molecules could directly dissolve cellulose without any processing. Therefore, these nano-composites prepared using CNTs and the cellulose dissolved with ionic liquid are expected to have widely potential applications.

Cellulose/MWCNT composite as a new type of material has unique structure and excellent physical and chemical 
properties. It combines the excellent performances of both cellulose (good biocompatibility) and CNTs (high surface area and strength), and also it is a novel functional and structural material. Cellulose/MWCNT composites have broad application prospect in the fields of biosensors and electrochemistry [10-14]. IL can directly dissolve cellulose and cannot affect the cooperative properties of the MWCNTs and cellulose. It is significant to reduce the costs and process steps of these composites. Carboxymethyl cellulose sodium (CMC) is a sodium salt of cellulose. As far as we know, the study of CMC/MWCNT biocomposites has not been previously reported.

In this paper, a biocompatible CMC/MWCNT biocomposite was synthesized using MWCNTs functionalized with CMC dissolved in IL. Especially, the electrocatalytic performance of the glass carbon electrode coated with $\mathrm{CMC} /$ MWCNT biocomposite towards $\mathrm{H}_{2} \mathrm{O}_{2}$ was measured. The effects of CNT content and ultrasonic time on the electrochemical performance were also studied.

The pristine MWCNTs used (average diameter: $11 \mathrm{~nm}$; purity: $\geqslant 95 \%$ ) came from Beijing Nano Technology Co., Ltd. (China). CMC was purchased from Tianjin Dongli Chemical Reagent Co., Ltd. (China). Concentrated sulfuric acid, concentrated nitric acid, pyridine, $n$-butane bromide and acetonitrile/ethyl acetate were supplied by National Medicine Inc. of Shaanxi Province. All the reagents were commercially available and of analytical grade. The chemicals were all used as received without further purification.

Carboxylic MWCNTs (f-MWCNTs) were prepared using a mixed solution $\left(\mathrm{HNO}_{3}: \mathrm{H}_{2} \mathrm{SO}_{4}=1: 3, \mathrm{v} / \mathrm{v}\right)$ by ultrasonication for $5 \mathrm{~h}$ at $60^{\circ} \mathrm{C}$. They were then rinsed with deionized water until the solution was neutral. Finally, they were baked in a vacuum oven at $100^{\circ} \mathrm{C}$ for $8 \mathrm{~h}$.

Preparation of IL: first, pyridine $(40.0 \mathrm{~g})$ was added into a $250 \mathrm{~mL}$ round-bottomed flask that was sealed with turned-mouth rubber stoppers. Then nitrogen was pumped and filled into the flask three times repeatedly. At room temperature and under magnetic stirring, $75.4 \mathrm{~g}$ of butane bromide was added into the flask using micro-syringe, and then heated to $50^{\circ} \mathrm{C}$ using water-bath apparatus and stirred for $12 \mathrm{~h}$. The product was added to $200 \mathrm{~mL}$ of ethyl acetate, the process of oscillation and washing operation were carried out five times. And then this product was put into 200 $\mathrm{mL}$ of acetonitrile for recrystallization. Finally, the product was dried in a vacuum oven at $80^{\circ} \mathrm{C}$ for $12 \mathrm{~h}$.

Preparation of CMC/MWCNT composite: first, $1 \mathrm{mg}$ of MWCNTs and $20 \mathrm{~g}$ of IL were added into a $100 \mathrm{~mL}$ round-bottomed flask under ultrasonication for $1 \mathrm{~h}$ at $80^{\circ} \mathrm{C}$. Then $20 \mathrm{~g}$ of IL containing $5 \mathrm{wt} \% \mathrm{CMC}$ was added to the above solution, and the mixture solution was sonicated for 1 $\mathrm{h}$, followed by adding $20 \mathrm{~mL}$ of deionized water. The reaction solution was filtrated with $0.22 \mu \mathrm{m}$ mixed-fiber membranes. The resulting black product was dispersed in deionized water again after leaching and repeated five times. Finally, the obtained CMC/MWCNT composites were distributed into $5 \mathrm{~mL}$ of deionized water.

A $10 \mathrm{mg} \mathrm{mL}^{-1} \mathrm{CMC} / \mathrm{MWCNT}$ water solution was prepared by ultrasonication for $10 \mathrm{~min}$. The GC electrode was polished using 0.3 and $0.05 \mu \mathrm{m}$ alumina slurry in sequence, rinsed with deionized water, and sonicated in acetone, ethanol and deionized water for $4 \mathrm{~min}$ each procedure. This solution was deposited on to the clean surface of GC electrode using a micro syringe, and finally dried with an infrared lamp to obtain a finished GC electrode coated with a CMC/MWCNT composite.

The X-ray diffraction (XRD) pattern was obtained on an X'Pert PRO diffractometer with Co K $\alpha$ radiation. The surface morphology of CMC/MWCNT composite was investigated by SUPRA $^{\mathrm{TM}} 55$ field-emission scanning electron microscopy (FESEM) at an accelerating voltage of $5 \mathrm{kV}$. High resolution transmission electron microscopy (HRTEM) was conducted with JEOL JEM-3010 at an accelerating voltage of $300 \mathrm{kV}$. Electrochemical performances were measured using a CHI 650C electrochemical workstation (CHI Instrument Company, Shanghai, China) with a threeelectrode system which composes of either a glass carbon electrode (GCE) or a GCE coated with a CMC/MWCNT composite as the working electrode, a saturated calomel electrode (SCE) as the reference electrode, and a Pt wire as the counter electrode.

Figure 1 shows the SEM images of f-MWCNTs (a) and CMC/MWCNT composites (b) (the content of MWCNTs is
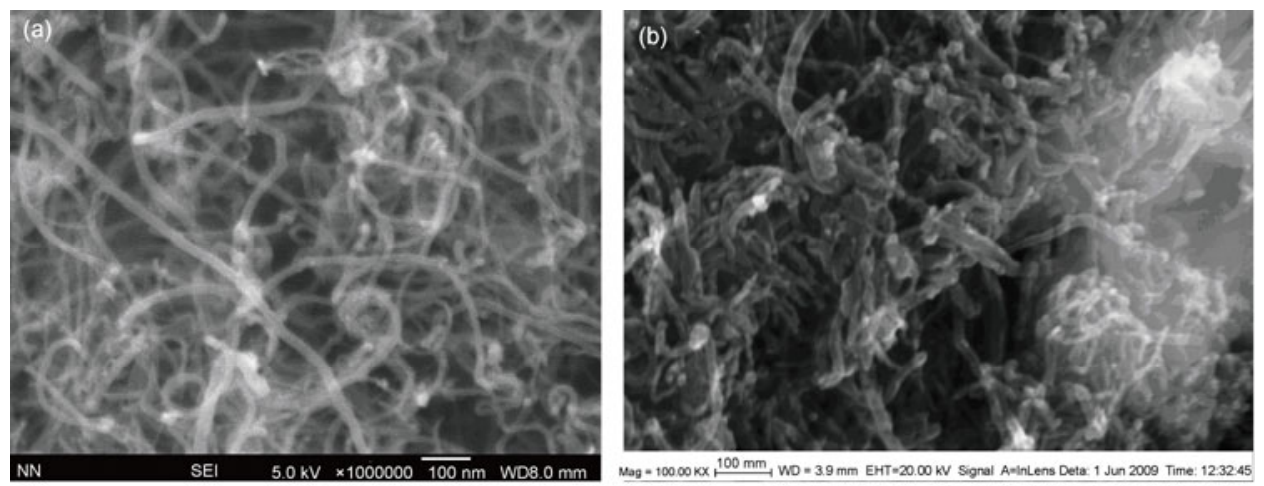

Figure 1 SEM images of f-MWCNTs (a) and CMC/MWCNT composite (b). 
$2 \mathrm{wt} \%$ and the ultrasonic time is $2 \mathrm{~h}$ ). It does not exist any coverings on the surface of f-MWCNTs and the tube-walls are smooth as shown in Figure 1(a). However, Figure 1(b) displays the morphology of CMC/MWCNT composites still exhibit the tubular shape. The tube-walls are rough due to the coated polymer on the surface of MWCNTs and the formed composites twisted each other. It indicates that the ultrasonic process improved the formation of $\mathrm{CMC} /$ MWCNT composite.

Figure 2 shows the HR-TEM images of f-MWCNTs (a) and $\mathrm{CMC} / \mathrm{MWCNT}$ composites (b). Figure 2(a) displays that the tube-wall of MWCNTs is smooth and thick. The graphite layers are clear and it does not exist amorphous carbon and catalyst particles. Figure 2(b) shows the uniform polymer coated on the surface of MWCNTs. The tube-wall structure of MWCNTs is compact and the polymer layers are loose. The mean thickness of CMC layer is about 5.4 nm. While CMC uniformly coated on the surface of MWCNTs, a core (MWCNTs)-shell (CMC) structure formed. The structure of CMC/MWCNT composite was clearly observed using HRTEM owing to the different densification of MWCNTs and CMC layers.

Figure 3 shows the XRD pattern of CMC/MWCNT

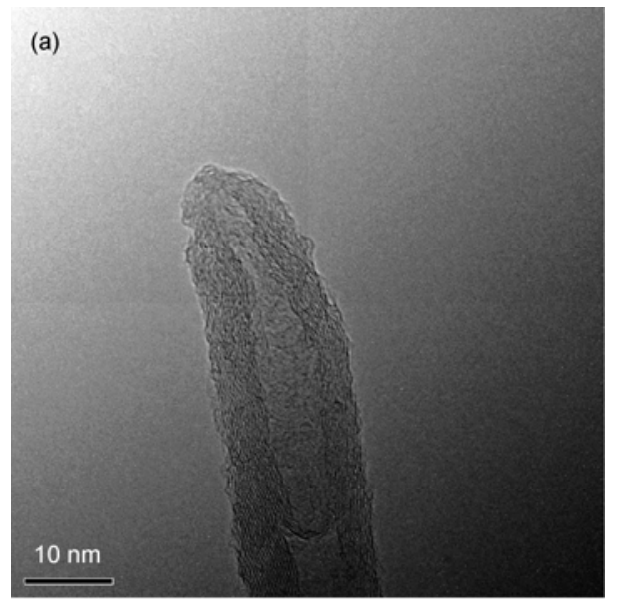

(b)

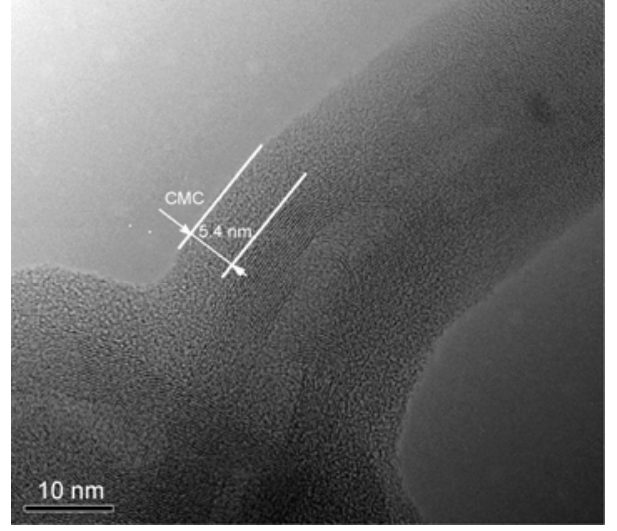

Figure 2 HR-TEM images of MWCNTs (a) and CMC/MWCNT composite (b).

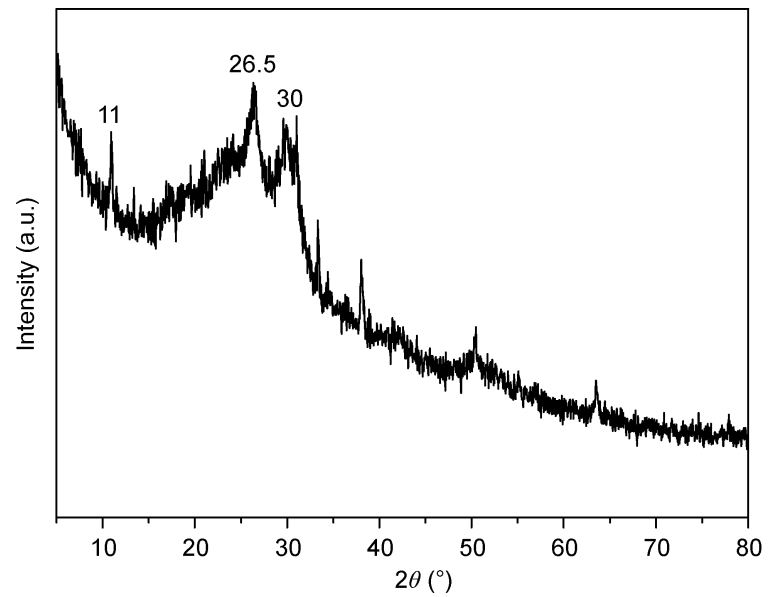

Figure 3 XRD pattern of CMC/MWCNT composite.

composite (the content of MWCNTs is $2 \mathrm{wt} \%$ and the ultrasonic process time is $2 \mathrm{~h}$ ). It indicates that there exist two sharp diffraction peaks at $11^{\circ}$ and $30^{\circ}$. Compared to CMC diffraction peaks, these peaks show that the composite contains a certain amount of CMC. Meanwhile, there exists a diffraction peak at $26.5^{\circ}$ and it is the characteristic peak of MWCNTs. It indicates that the CMC has formed in the composite in agreement with Figure 2. XRD results indicate CMC has been successfully coated on the surface of MWCNTs.

Figure 4 shows the effect of ultrasonic time on the dispersivity of CMC/MWCNTs in water solution. It indicates that the ultrasonic time increasing can improve the combination between CMC and MWCNTs. The dispersivity of CMC/MWCNT composite in water solution with ultrasonic time of $2 \mathrm{~h}$ is better than that of ultrasonic time 0.5 and $1 \mathrm{~h}$. It remains in uniformly dispersed state after standing for $72 \mathrm{~h}$ at room temperature. The agglomerated and precipitated materials were found under the ultrasonic time of 0.5 and $1 \mathrm{~h}$, especially, the precipitation is obvious under the ultrasonic time of $0.5 \mathrm{~h}$. MWCNTs coated with CMC dispersed uniformly in water solution because CMC contains a large amount of polar groups such as $\mathrm{OH}^{-}$which easily occur hydrophilic interaction. The dispersivity of CMC/MWCNTs in water solution was improved effectively by using ultrasonic treatment.

$\mathrm{H}_{2} \mathrm{O}_{2}$ is used as a raw material or intermediate product in industrial process, and a by-product of oxidase reaction in living organisms. So it is very important to detect the content of $\mathrm{H}_{2} \mathrm{O}_{2}$ in food, medicine, industry and environmental analysis. Electrochemical method is widely applied for the detection of electrochemical active material because of its high sensitivity, wide linear-range, and fast response. The electrochemical property of the GCE coated with a CMC/MWCNT composite in $\mathrm{H}_{2} \mathrm{O}_{2}$ phosphate buffer solution (PBS) is discussed.

Figure 5 shows cyclic voltammograms (CVs) of the different electrodes in $10 \mathrm{mmol} / \mathrm{L} \mathrm{H}_{2} \mathrm{O}_{2} / \mathrm{PBS}$ solution ( $\mathrm{pH}$ 6.8) 
(a)

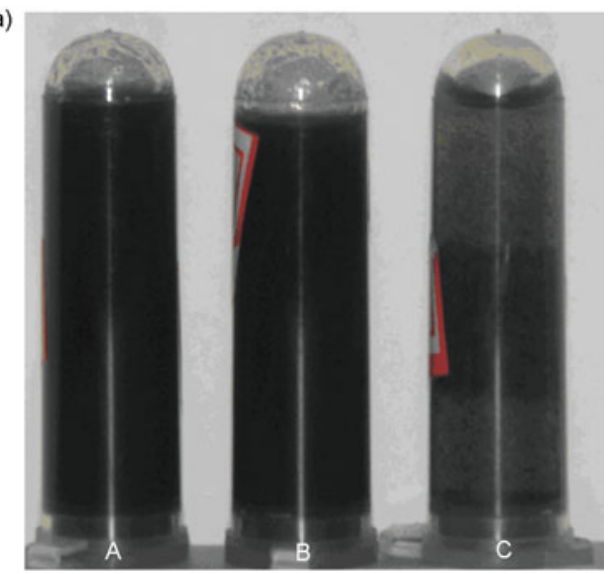

(b)

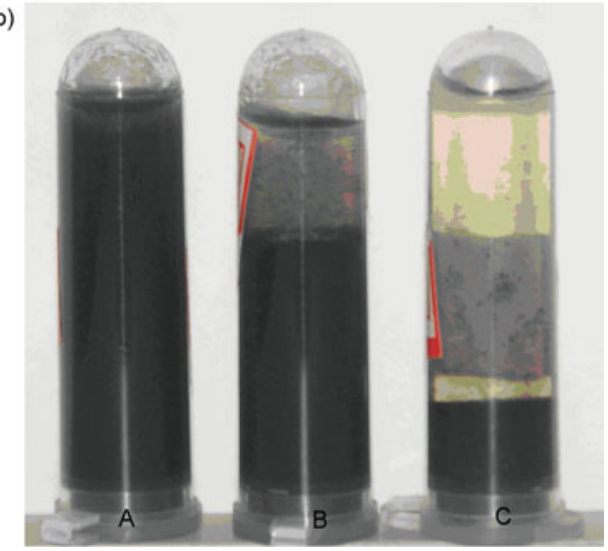

Figure 4 (Color online) The dispersivity of CMC/MWCNTs in water solution after standing for $40 \mathrm{~min}(\mathrm{a}) ; 72 \mathrm{~h}$ (b). Ultrasonic time: A, 2; B, 1; $\mathrm{C}, 0.5 \mathrm{~h}$.

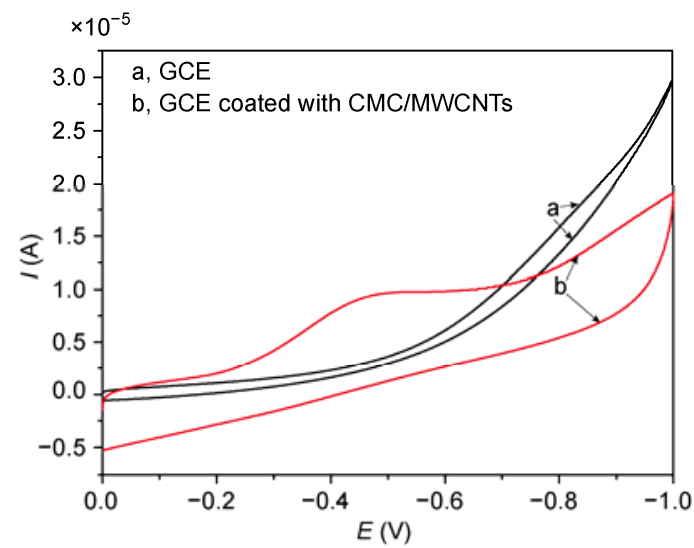

Figure 5 (Color online) CVs of the different electrodes: a, GCE; b, GCE coated with $\mathrm{CMC} / \mathrm{MWCNT}$ composite in $10 \mathrm{mmol} / \mathrm{L} \mathrm{H}_{2} \mathrm{O}_{2} / \mathrm{PBS}$ solution.

(scan rate: $0.04 \mathrm{~V} / \mathrm{s}$ ). There does not exist a redox peak in the curve of bare GCE, however, the CV curve of GCE coated with a CMC/MWCNT composite shows an obvious oxidation peak at $-0.47 \mathrm{~V}$. One possible explanation is that the CMC/MWCNT composite contains plenty of MWCNTs and CMC. MWCNTs can accelerate the electron exchange of electrochemical active material due to its excellent elec- trocatalytic characteristics. Furthermore, some active groups on CMC have the function of absorption, chelation and exchange. The superficial porous structures of CMC/MWCNT composite change the superficial structure of this electrode, and it is propitious for the enrichment of substance and the diffusion of micromolecule. Therefore, the electrocatalytic property of GCE coated with a CMC/MWCNT composite has been improved significantly.

Figure 6 shows CVs of the GCE coated with $\mathrm{CMC}$ / MWCNTs in $10 \mathrm{mmol} / \mathrm{L} \mathrm{H}_{2} \mathrm{O}_{2} / \mathrm{PBS}$ solution ( $\mathrm{pH} \mathrm{6.8)} \mathrm{(scan}$ rate: $0.04 \mathrm{~V} / \mathrm{s}$ ) with different solvents (deionized water and self-made IL). It indicates that there does not exist a redox peak in CV of the GCE coated with CMC/MWCNT composite in deionized water. However, there exists an obvious oxidation peak at $-0.47 \mathrm{~V}$ in the $\mathrm{CV}$ of the GCE coated with CMC/MWCNT composite in IL. This is because IL has a high polarity and can fully dissolve CMC. The dissolved CMC wrapped on the surface of MWCNTs to form a good functional CMC/MWCNT composite.

Figure 7 presents CVs of the GCE coated with a CMC/ MWCNT composite at different content of MWCNTs. It shows that the oxidation peak is obvious while the content of MWCNTs is $3 \mathrm{mg}$ (i.e. $2 \mathrm{wt} \%$ content). However, while the content of MWCNTs is 1 and 5, 2 and $4 \mathrm{mg}$ respectively, the CVs of the GCE coated with CMC/MWCNT composite are similar, and the oxidation peaks are not evident in these curves. This is because both MWCNTs and CMC play an equal and vital role in the formation of the composites. While the content of MWCNTs is too low, CMC firmly coats on the surface of MWCNTs and fills into the porous intervals between MWCNTs. The dense coating greatly induces the electrocatalytic property of the composites. Otherwise, as the content of MWCNTs is too high, the CMC coated on MWCNTs is not enough to improve the dissolvability and dispersibility of MWCNTs. So the optimal content of MWCNTs in the composite is $2 \mathrm{wt} \%$.

Figure 8 shows $\mathrm{CVs}$ of the GCE coated with $\mathrm{CMC}$ / MWCNT composite at different ultrasonic time. The oxidation peak is obvious under the ultrasonic time of $2 \mathrm{~h}$. How-

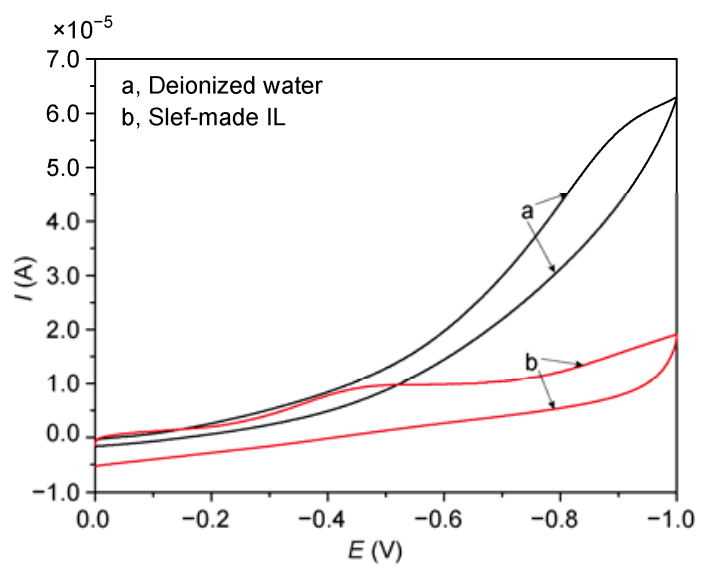

Figure 6 (Color online) CVs of GCE coated with CMC/MWCNT composite in different solvents: a, deionized water; b, self-made IL. 


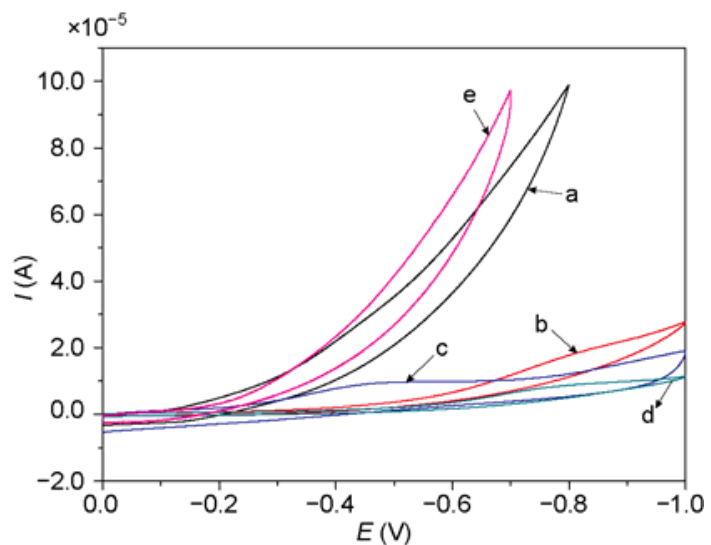

Figure $7 \mathrm{CVs}$ of GCE coated with CMC/MWCNT composite in 10 $\mathrm{mmol} / \mathrm{L} \mathrm{H}_{2} \mathrm{O}_{2} / \mathrm{PBS}$ ( $\mathrm{pH} 6.8$ ) solution (ultrasonic time: $2 \mathrm{~h}$; scan rate: 0.04 $\mathrm{V} / \mathrm{s}$ ) at different contents of CNTs: a, 1; b, 2; c, 3; d, 4; and e, $5 \mathrm{mg}$.

ever, the CVs of the GCE coated with CMC/MWCNT composite at the ultrasonic time of $1,1.5,2.5$ and $3 \mathrm{~h}$ is similar, and the oxidation peaks are not evident. The possible reason is that CMC would be fully dissolved with increasing ultrasonic time so that they filled into the porous intervals between MWCNTs, and firmly coated on the surface of MWCNTs. The results lead to the formation of dense composites, which restrict $\mathrm{H}_{2} \mathrm{O}_{2}$ diffusion. The active groups (e.g. epoxy \& carboxymethyl) on CMC were destroyed at a long ultrasonic time, so it affected the electrocatalytic property of the composites. Otherwise, CMC cannot be fully dissolved while the ultrasonic time is very short. The electrochemical activity of CMC/MWCNTs is weak due to the low content of CMC coated on the surface of MWCNTs. The sonication treatment plays an important role in the dissolvability and dispersibility of CMC. It can greatly affect the electrocatalytic property of the GCE coated with a CMC/MWCNT composite.

Figure 9 shows $\mathrm{CV}$ s of the GCE coated with $\mathrm{CMC} /$ MWCNT composite in $10 \mathrm{mmol} / \mathrm{L} \mathrm{H}_{2} \mathrm{O}_{2} / \mathrm{PBS}$ at different scan rates. The oxidation peak currents improved with the

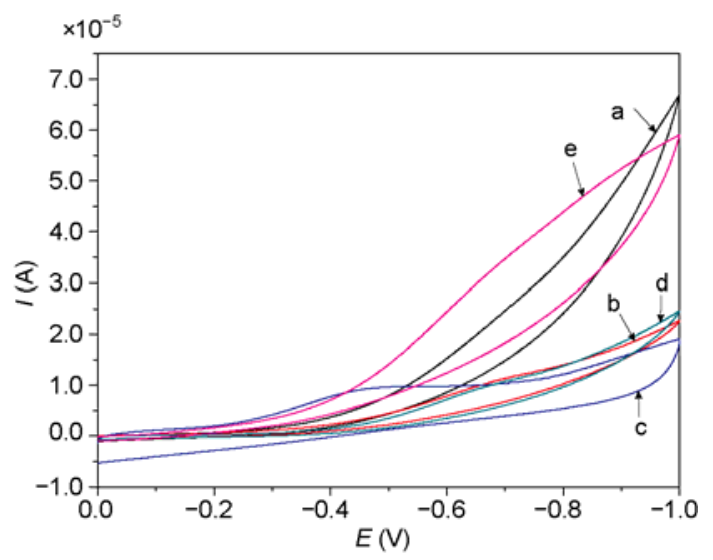

Figure $8 \mathrm{CVs}$ of the GCE coated with CMC/MWCNT composite in 10 $\mathrm{mmol} / \mathrm{L} \mathrm{H}_{2} \mathrm{O}_{2} / \mathrm{PBS}$ solution ( $\mathrm{pH} 6.8$ ) (CNTs: $2 \mathrm{wt} \%$; scan rate: $0.04 \mathrm{~V} / \mathrm{s}$ ) at different ultrasonic time: $\mathrm{a}, 1.0 ; \mathrm{b}, 1.5 ; \mathrm{c}, 2.0 ; \mathrm{d}, 2.5 ; \mathrm{e}, 3.0 \mathrm{~h}$.

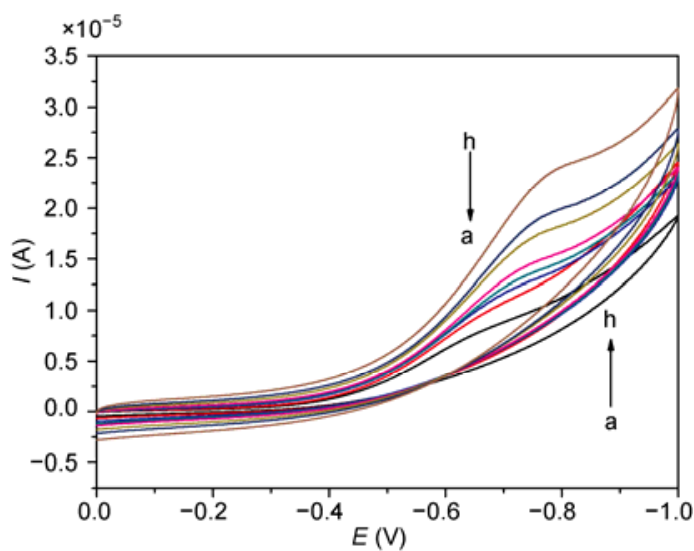

Figure $9 \mathrm{CVs}$ of GCE coated with CMC/MWCNT composite in 10 $\mathrm{mmol} / \mathrm{L} \mathrm{H}_{2} \mathrm{O}_{2} / \mathrm{PBS}$ solution ( $\mathrm{pH}$ 6.8) (CNTs: $2 \mathrm{wt} \%$; ultrasonic time: $2.5 \mathrm{~h}$ ) at various scan rates: a, $0.02 ; \mathrm{b}, 0.04 ; \mathrm{c}, 0.06 ; \mathrm{d}, 0.08 ; \mathrm{e}, 0.1 ; \mathrm{f}, 0.15 ; \mathrm{g}, 0.2$; and h, $0.3 \mathrm{~V} / \mathrm{s}$

increasing of scan rate, and the peak current was up to a higher value at a scan rate of $0.04 \mathrm{~V} / \mathrm{s}$. It implied that the GCE coated with CMC/MWCNT composite has a good eletrocatalytic effect towards $\mathrm{H}_{2} \mathrm{O}_{2}$.

In summary, water-soluble CMC/MWCNT composites were prepared using MWCNTs modified with a CMC dissolved in IL prepared by a sealed water-bath heating apparatus. When the content and ultrasonic processing time of MWCNTs was $2 \mathrm{wt} \%$ and $2 \mathrm{~h}$ respectively, CMC could be more evenly wrapped on the surface of MWCNTs and the coating thickness of CMC was about $5.4 \mathrm{~nm}$. The dispersivity and electrochemical properties of CMC/MWCNT composite were affected by ultrasonic time. IL played a vital role in the preparation of CMC/MWCNT composite. The GCE coated with CMC/MWCNT composite presented good electrocatalytic properties towards $\mathrm{H}_{2} \mathrm{O}_{2}$ because the surface structure of MWCNTs enwrapped with CMC was changed. Especially, the electrocatalytic effect of GCE coated with a CMC/MWCNT composite was greatly affected by the MWCNT content and ultrasonic treatment time. When the content of MWCNTs was $2 \mathrm{wt} \%$ and the ultrasonic time was $2 \mathrm{~h}, \mathrm{CMC} / \mathrm{MWCNT}$ composite had the best electrochemical performance towards $\mathrm{H}_{2} \mathrm{O}_{2}$.

This work was supported by the Specialized Research Fund for the Doctoral Program of Higher Education of China (20096102120016, 20116102110014), the National Natural Science Foundation of China (51172184), the Innovation Foundation of China Aerospace Science and Technology (CASC200906), the Natural Science Foundation of Shaanxi Province (2009GM6001-1) and the Foundation for Fundamental Research of Northwestern Polytechnical University (JC201030).

1 Iijima S. Nature, 1991, 354: 56-58

2 Esawi A M K, Farag M M. Mater Des, 2007, 28: 2394-2401

3 Kocharova N, Leiro J, Kankare J, et al. Langmuir, 2007, 23: 3363-3371

4 Edgar K J, Buchanan C M, Debenham J S, et al. Prog Polym Sci, 2001, 26: 1605-1688 
5 Yun S, Kim J. Sensor Actuat B, 2010, 150: 308-313

6 Fukushima T, Kosaka A, Ishimura Y, et al. Science, 2003, 300: 2072-2074

7 Welton T. Chem Rev, 1999, 99: 2071-2082

8 Jonathan G H, Heather D W, Richard P S, et al. Chem Commun, 1998, 1765-1766

9 Swatloski R P, Spear S K, Holbrey J D, et al. J Am Chem Soc, 2002,
124: 4974-4975

10 Tian R, Li S Q, Wan L, et al. Chem Ind Eng Prog, 2008, 27: 1569-1573

11 Fu C L, Meng L J, Lu Q H. J Mater Eng, 2008, (8): 26-30

12 Nadagouda M N, Varma R S. Macromol Rapid Commun, 2007, 28: 842-847

13 Fukushima T, Aida T. Chem Eur J, 2007, 13: 5048-5058

14 Marcilla R, Curri M L, Cozzoli P D, et al. Small, 2006, 2: 507-512

Open Access This article is distributed under the terms of the Creative Commons Attribution License which permits any use, distribution, and reproduction in any medium, provided the original author(s) and source are credited. 